\title{
A Study of Biocompatibility of Acellular Matrices of Primate Lungs and the Potential tor Their Application as Tissue- Engineering Constructions
}

DOI: $10.17691 / \mathrm{stm} 2017.9 .4 .10$

Received April 21, 2017

E.V. Kuevda, MD, PhD, Researcher, Laboratory of Fundamental Research in the Field of Regenerative Medicine",

E.A. Gubareva, MD, PhD, Head of the Laboratory of Fundamental Research in the Field of Regenerative Medicine';

1.S. Gumenuyk, Researcher, Laboratory of Fundamental Research in the Field of Regenerative Medicine';

D.D. Karal-ogly, PhD, Head of the Laboratory of Preclinical and Clinical Studies of Pharmaceuticals and Medical Products ${ }^{2}$

TKuban State Medical University, Ministry of Health of the Russian Federation, 4 Sedina St., Krasnodar, 350063, Russian Federation;

${ }^{2}$ Research Institute of Medical Primatology, 177 Mira St., Vesyoloe, Sochi, Adler Region, 354376, Russian Federation

The aim of the study was to evaluate biocompatibility of acellular matrices on the model of primate lungs, which is closely approximated to human lungs in terms of anatomic-morphological characteristics, for the purpose of creating tissue engineering constructions.

Materials and Methods. Acellular matrix was obtained from detergent-enzymatic decellularization of primate lungs according to a modified protocol, where exposition time and detergent concentration were increased. The quality of the obtained scaffold was morphologically evaluated after staining with hematoxylin and eosin and DAPI fluorophore, and by testing the assay for residual DNA. The change in the composition of decellularized extracellular matrix was quantitatively evaluated during the registration of the square area of positive staining after immunohistochemical testing and during staining with alcian blue. Biocompatibility of scaffolds was tested with XTT assay after 48 and $72 \mathrm{~h}$ of static recellularization with a consequent quantitative determination. As the main cellular resource for colonization of the scaffolds, we used multipotent mesenchymal stem cells of primates whose cellular affiliation was verified by immunophenotyping and targeted differentiation.

Results. Morphological testing did not reveal any considerable structural damage of the scaffolds, residual DNA was $19.2 \%$. Quantitation of the square area of positive staining revealed a statistically significant decrease in the amount of type I collagen and the vascular endothelium growth factor (VEGF) after decellularization. Quantitative changes in extracellular matrix were registered, however they do not impair biocompatibility of the scaffolds and do not affect cell activity.

Conclusion. The obtained scaffolds do not have cytotoxic properties, they contribute to adhesion, growth and metabolic activity of cells, which allows us to consider acellular matrices to be a potential for creating tissue engineering constructions of lungs after additional enhancement of the protein composition.

Key words: acellular matrices; tissue-engineered constructions; biocompatibility of acellular matrices of lungs; detergent-enzymatic decellularization; recellularization.

The last decade is characterized by a rapid growth of allergic and infection pathologies as well as malignant neoplasms of the respiratory system. This trend along with the rise of cardiovascular diseases becomes socially important [1]. The existing methods of treatment, including organ transplantation, cannot completely solve the problem of combating chronic respiratory diseases. For this reason, regenerative medicine is of particular interest because it can offer potentially new methods of treatment. For example, tissue engineering offers the use of recellularized biological scaffolds of lungs obtained by decellularization from donors suitable not in all parameters as an ex vivo model of lung regeneration. A decrease in immunogenicity of the scaffolds and absence of need for constant immunosuppressive therapy after transplantation can be achieved by recellularization with the recipient's own stem cells or endogenic progenitor cells [2]. Moreover, the organs after decellularization can be a potential model for studying interaction of cells and extracellular matrix in pathological changes in lungs like emphysema or fibrosis as well as in study of age changes [3, 4].

For contacts: Elena V. Kuevda, e-mail: elenakuevda@yandex.ru 
Biological scaffolds obtained from small laboratory animals are widely used as a model for developing protocols of decellularization and recellularization and study of biocompatibility of matrices and intercellular interaction $[5,6]$. However the results obtained on rodent models cannot always be optimally and without quality losses applied to bigger animals due to considerable interspecific and physiological differences as well as technical difficulties $[7,8]$. This leads to the need for modification of protocols of organ decellularization when working with big animals with further evaluation of the quality of the obtained scaffolds and ability to maintain adhesion, growth and proliferation of cells.

The aim of the study was to investigate biocompatibility of acellular matrix using the model of primate lungs, which are closely approximated to human lungs in terms of anatomic-morphological characteristics. The matrix was obtained as a result of decellularization using detergent-enzymatic method. We also aimed to assess the potential of using the proposed protocol for creating tissue engineering constructions.

\section{Materials and Methods}

Obtaining of acellular scaffold of primate lungs. For decellularization we used 3 organ-complexes "heartlung" of adult male rhesus monkeys (Macaca mulatta). The extraction of organs was performed at the Research Institute of Medical Primatology (Sochi) during autopsy after the natural death of the animals. The organs were rinsed in isotonic solution of sodium chloride and placed into a sterile phosphate buffer where $1 \%$ penicillinstreptomycin was added (Thermo Fisher Scientific Inc., USA). The organ-complexes were transported in a special container at the temperature not higher than $4^{\circ} \mathrm{C}$ to the Laboratory of Fundamental Research in the Field of Regenerative Medicine in Kuban State Medical University (Krasnodar) where decellularization of scaffolds with further evaluation of quality of the obtained matrix was performed. Transportation time did not exceed $24 \mathrm{~h}$. All procedures were performed in accordance with the "Regulations for Work with Experimental Animals" (Order of the Ministry of Health of the USSR No.755 dated August 22, 1972) and the European Convention for the Protection of Vertebrate Animals used for Experimental and Other Scientific Purposes (Strasbourg, 1986) after the study protocol had been approved by the local Ethics Committee (protocol No.30/1 dated 2014). For the development of lung decellularization of non-human primate lungs detergent-enzymatic method of exposure was used as a basic one, which had been earlier successfully modified and applied by us to obtain a biological scaffold of rat lungs [6]. The modification involved an increase in exposition time and concentration of the solution of sodium deoxycholate, as well as the method of injecting the decellularized agents. Importantly, it seemed impossible to use Harvard Inspira Advanced Safety Ventilator (Harvard Apparatus, USA), which is designed for rodents, as it would have been ineffective in providing the required parameters of the respiratory volume and vital capacity of the lungs due to greater weight of the animals. For this reason, perfusion with detergents was additionally done through trachea with natural outflow under constant control of the rate and volume of the incoming solution in order to avoid alveoli damage. The concentration of sodium deoxycholate was increased to $2 \%$. However supplementary methods of physical decellularization (for example, freezing with further gradual melting) were rejected during the preliminary evaluation of the scaffold quality due to the evident damaging effect [9]. The total length of exposure to detergents and enzymes was $48 \mathrm{~h}$.

Evaluation of quality and biocompatibility of acellular matrix of primate lungs. Initial morphological assessment of the quality of the obtained acellular matrices was performed using standard methods of histological staining with hematoxylin and eosin and DAPI fluorophore (4',6-diamidino-2phenylindole) in order to evaluate preservation of the scaffold architectonics and the effectiveness of nuclear material elimination. Quantification of residual DNA was performed with a colorimetric method in the spectrophotometer NanoDrop ND1000 (Thermo Fisher Scientific Inc., USA) [6, 9, 10]. Quantification of the amount of extracellular matrix components (glycosaminoglycans, collagen fibers and vascular endothelium growth factor (VEGF)) before and after decellularization was performed with the assay of the square area of stained parts of the samples in a narrow spectral region with optical density higher than the threshold according to the principle of color deconvolution [11, 12]. Preliminary preparation of samples involved staining with alcian blue for quantification of glycosaminoglycans and an immunohistochemical test on the amount of collagen and VEGF. As primary antibodies we used polyclonal antibodies in dilution 1:100 to type I collagen (ab 34710; Abcam, USA) and VEGF (ab 46154; Abcam, USA), respectively. Microphotos for further morphometric analysis were obtained with a microscope Olympus IX51 with camera Olympus XC30 (Olympus Corporation, Japan). The images were stored in tif format with the size $2,080 \times 1,544$ pixels. To analyze microphotos we chose random fields of vision without artefacts and edge defects for native and decellularized samples (for each of six samples at least 12 fields of vision). The obtained microphotos were processed in Rawtherapee software to normalize white-balance and exposition. A comparative morphometric analysis was performed with the software Morphostain developed by us [https:// github.com/meklon/morphostain; published under free license GNU GPL v. 3.0]. During the analysis we isolated the channels of individual stains using the method of color deconvolution [12]. After stain segmentation, the areas with color intensiveness exceeding the threshold limit were automatically identified and then the square area of stained parts was calculated. 
Biocompatibility of scaffolds was tested by XTT assays in 48 and $72 \mathrm{~h}$ of cell cultivation in acellular matrix. Recellularization of test samples $(n=6)$ was performed in static conditions in 96-well plates. Cell suspension in volume 100,000 cells per $200 \mu \mathrm{l}$ of culture medium was applied directly on the scaffold surface. As a positive control $(n=6)$ we used the cells cultivated on plastic without a biological scaffold; as a negative control $(n=6)$ and to exclude background effect of biological matrix on optical density we used samples of acellular matrix of lungs uncultivated with cells. As a cell resource, we selected multipotent mesenchymal stem cells of rhesus monkeys of the $6-7^{\text {th }}$ passages. Cell affiliation of the cells used for recellularization was verified with immunophenotyping using the method of flow cytofluorometry and targeted differentiation in three cellular lines. To study surface cell markers we used antibodies to CD34 (Lot 3277660), CD45 (Lot 3095590), CD73 (Lot 2237759), CD90 (Lot 2296945), CD105 (Lot 31516310) (Beckman Coulter, USA). Typing of cell lines was performed twice: in the primary culture and in the fifth passage with flow cytofluorometer Beckman Coulter (USA) at the Research Institute of Medical Primatology (Sochi). The induced differentiation was performed according to the standard method of using StemPro medium (Thermo Fisher Scientific Inc., USA) and the technology of staining with Oil Red $\mathrm{O}$ (evaluation of adipogenic differentiation), toluidine blue (evaluation of chondrogenic differentiation) and alizarin red (evaluation of matrix mineralization) which had been applied by us earlier [6].

Statistic processing of data. Statistic processing of data was performed using the methods of variation statistics on a personal computer with GraphPad Prism software v. 6.04 (source - www.graphpad.com). The obtained results are represented as $M \pm m$, where $M$ is an arithmetic mean, $m$ is the standard error of the mean. The probability of an error was determined according to Student's t-distribution table for paired comparison. For a selected sample different from the compared ones we used Mann-Whitney criterion. The differences were considered statistically significant at $p<0.05$.

Results. Routine methods of staining samples of primate lungs confirmed complete absence of nuclei and cell elements. Damage of alveolar structure and architectonics of matrix in general are expressed insignificantly (Figure 1). Quantification of residual DNA after decellularization was $148.3 \pm 16.22 \mathrm{ng} / \mathrm{mg}$ of tissue $(19.2 \%)$, while in native lungs it was equal to $771.2 \pm 67.99 \mathrm{ng} / \mathrm{mg}$ of tissue $(p=0.0001)$. Positive staining of sulfated glycosaminoglycans with alcian blue was marked both on native lungs sections and on decellularized samples. At the same time, quantitative recalculation of positive staining showed a decrease in the level of glycosaminoglycans after exposure to detergents. The tendency of the amount of extracellular components to decrease, was also noted during quantitative calculation of total square area of positive
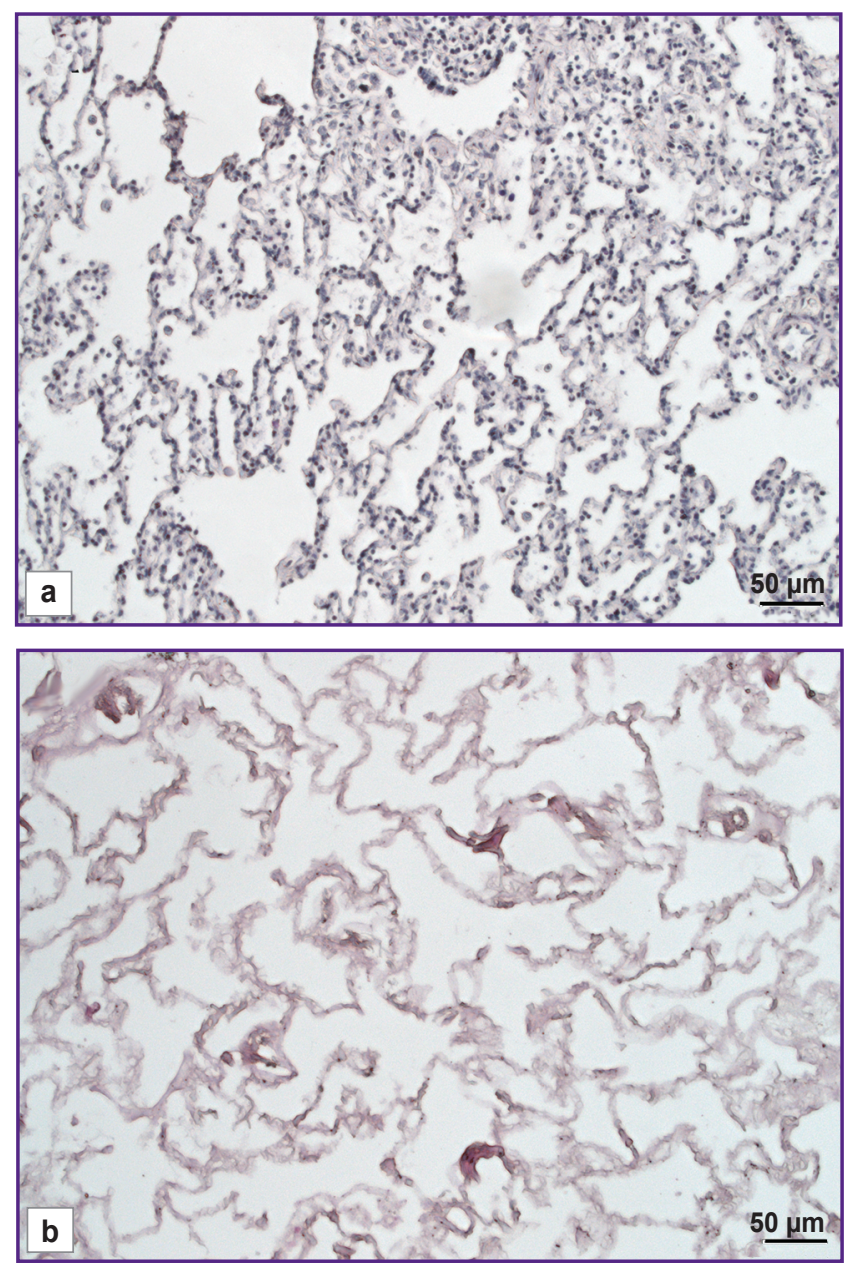

Figure 1. Morphological characteristics of alveolar structure of primate lungs before (a) and after (b) decellularization; staining with hematoxylin and eosin; lens magnification $\times 20$; ocular magnification $\times 10$

staining for VEGF, but statistically relevant changes in the amount of type I collagen fibers were not identified $(p=0.1595$, Mann-Whitney criterion) (Figure 2). This is consistent with the previously obtained data [11] on decrease in the amount of elastin after the end of lung decellularization due to the damaging effect of detergents.

As a result of immunophenotyping of cells, we identified lack of CD34 and CD45 expression and positive expression of CD73, CD90 and CD105. Quantitative distribution of cells depending on the presence of surface markers is represented in Table.

The effect of specialized induction media on cell culture contributes to targeted adipogenesis, chondrogenesis and osteogenesis, which was qualitatively confirmed by presence of positive staining. This leaves no doubt that the used culture belongs to multipotent mesenchymal stem cells.

The results of XTT assays on the second and third days of cultivation showed that the scaffold is not toxic, 
Figure 2. Quantitative characteristics of protein content of extracellular matrix of native and decellularized primate lungs:

GAG NL and GAG_DL - the amount of glycosaminoglycans in native and decellularized lungs; Col 1_NL and Col 1_DL - the amount of type I collagen in native and decellularized lungs; VEGF_NL and VEGF_DL - the amount of VEGF in native and decellularized lungs; * $p=0.0001$ contributes to adhesion, growth maintenance and metabolic activity of cells (Figure 3 ).

Discussion. The detergent-enzymatic method of decellularization of native organs is widely used to obtain acellular matrices for tissue engineering. Translation of methods of decellularization of small laboratory animals to larger models requires considerable modification of the protocols of obtaining biological scaffolds and methods of evaluation of quality and biocompatibility of matrices. A number of leading scientists in the USA and Western Europe [1315] offer some protocols of obtaining acellular matrix of swine and primate lungs. However, those protocols involve long processing of native organs with detergent solutions (up to 3-7 days) [13-15], which potentially can cause bacterial and viral contamination of the obtained material. Effectiveness of using buffered $10 \%$ formalin as a preservative for further recellularization of matrix is also doubtful [13] because of toxicity of the applied method of preliminary processing. Furthermore, a number of scientists use aggressive solutions of detergents, such as sodium dodecyl sulfate, which results in practically complete destruction of laminin fibers and type I collagen and in explicit decrease in elastin and type IV collagen in extracellular matrix [15]. The study of cytotoxicity of the obtained scaffolds was performed by foreign authors [13, 15] only by registering proliferative activity of cells on the scaffold during cultivation which takes at least 7 days. The use of MTT assay to evaluate the capacity of cells to proliferate on decellularized scaffolds [14] is limited by toxicity of reagents which leaves room for the development of new methods of evaluation of cytotoxic properties of acellular matrices.

The suggested here use of sodium salt of

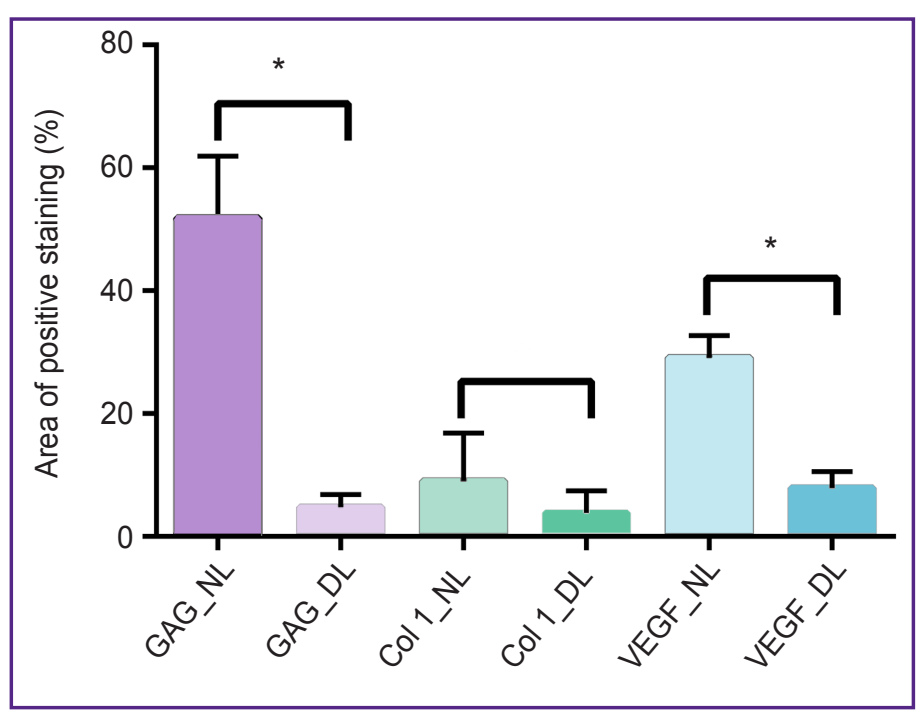

Immunophenotyping of multipotent mesenchymal stem cells (MSCs) of primates

\begin{tabular}{|c|c|c|c|c|c|c|}
\hline \multirow{2}{*}{$\begin{array}{l}\text { Number } \\
\text { of the typed } \\
\text { sample }\end{array}$} & \multirow{2}{*}{ Sample type } & \multicolumn{5}{|c|}{$\begin{array}{l}\text { Results of expression } \\
\text { of surface markers (\%) }\end{array}$} \\
\hline & & CD45- & CD90+ & CD34- & CD105+ & CD73+ \\
\hline \multirow{2}{*}{1} & Primary culture & 32.5 & 51.7 & 30.0 & 49.3 & 55.6 \\
\hline & MSCs, passage 5 & 29.8 & 28.4 & 38.6 & 31.5 & 32.7 \\
\hline \multirow{2}{*}{2} & Primary culture & 52.0 & 54.6 & 26.4 & 55.4 & 56.1 \\
\hline & MSCs, passage 5 & 31.4 & 28.4 & 32.8 & 24.7 & 28.6 \\
\hline \multirow{2}{*}{3} & Primary culture & 28.6 & 52.6 & 25.5 & 54.6 & 57.1 \\
\hline & MSCs, passage 5 & 24.9 & 33.9 & 33.3 & 31.1 & 32.0 \\
\hline
\end{tabular}

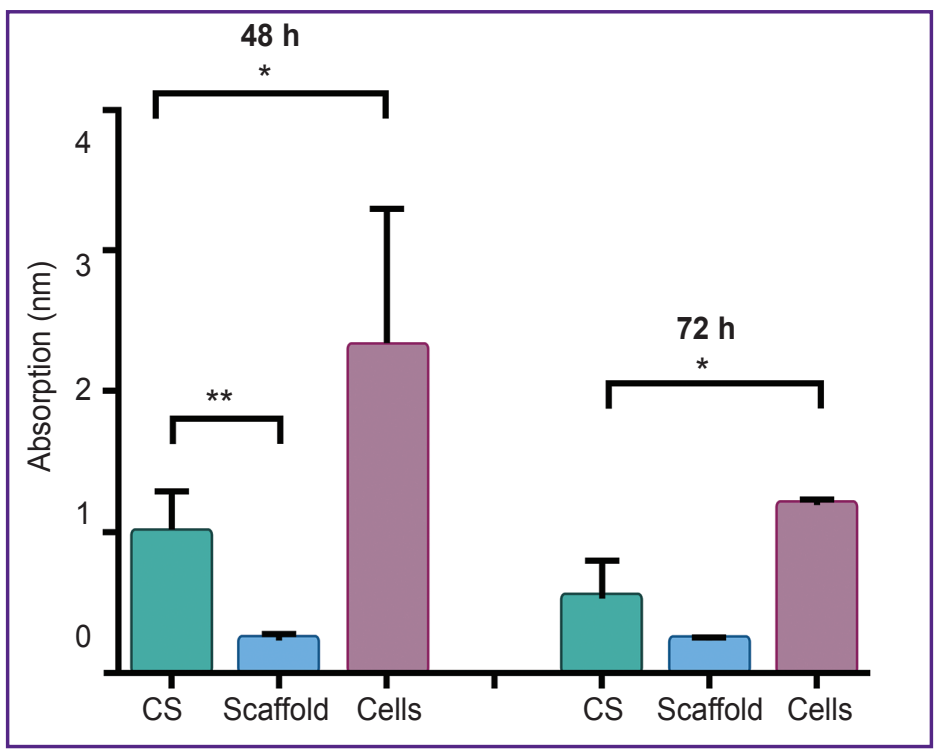

Figure 3. The results of scaffold compatibility tests in 48 and $72 \mathrm{~h}$ of cultivation:

CS is cells on acellular scaffold; scaffold is uncultivated decellularized matrix; cells are control cells cultivated on plastics; ${ }^{*} p \leqslant 0.05$; ${ }^{* *} p=0.0016$ 
deoxycholic acid as the main detergent, increase in the time of solution exposition and increase in their concentration contribute to a complete elimination of foreign cellular material without explicit structural damage of extracellular matrix. Total time of exposure to decellularized agents was lowered to $48 \mathrm{~h}$, which led to a reduced risk of contamination. During staining with alcian blue with further calculation of the square area of positive staining of the sample of native and decellularized primate lungs we registered considerable quantitative changes in the composition of extracellular matrix (decrease in the amount of sulfated glycosaminoglycans after decellularization) which were impossible to determine when the same sample was stained with alcian blue and the results were visually evaluated. The identified changes do not worsen biocompatibility of scaffolds and do not have an impact on cellular activity, though they reduce the compliance of lung tissue after decellularization. XTT assay used by the authors to evaluate metabolic activity and vitality of cells on scaffolds allowed us to assess cytotoxic properties of the scaffolds within the shortest term possible. The obtained matrices did not have toxic properties and did not suppress proliferative and metabolic activity of cells.

Conclusion. The performed studies on obtaining and investigating biocompatibility of acellular matrices of primate lungs are an important step towards creation of three-dimensional biological scaffolds. The lack of cytotoxic properties of decellularized matrices, their capacity to maintain cell growth and create proper microenvironment on the model of primate lungs closely approximated to human in their anatomic-morphological qualities, suggest potential possibility of clinical use of tissue-engineering construction of lungs.

Financing of the Study. The study was performed under the complex scientific research work "Cellular Mechanisms of Regeneration of Intrathoracic Organs and Tissues. The development of tissue-engineering constructions with the use of biological and synthetic scaffolds".

Conflict of Interests. The authors have no conflict of interests to declare.

\section{References}

1. Ignatova G.L., Zakharova I.A., Sarsenbayeva A.S., Rodionova O.V., Grebneva I.V., Drozdov I.V., Blinova E.V., Minakina O.L., Antonov V.N., Belsner M.S., Pustovalova I.A. Features of chronic bronchitis in different age groups. International Journal of BioMedicine 2014; 4(1): 15-18.

2. Wagner D.E., Bonvillain R.W., Jensen T., Girard E.D., Bunnell B.A., Finck C.M., Hoffman A.M., Weiss D.J. Can stem cells be used to generate new lungs? Ex vivo lung bioengineering with decellularized whole lung scaffolds. Respirology 2013; 18(6): 895-891, https://doi.org/10.1111/ resp.12102.

3. Sokocevic D., Bonenfant N.R., Wagner D.E., Borg Z.D., Lathrop M.J., Lam Y.W., Deng B., Desarno M.J., Ashikaga T., Loi R., Hoffman A.M., Weiss D.J. The effect of age and emphysematous and fibrotic injury on the re-cellularization of de-cellularized lungs. Biomaterials 2013; 34(13): 3256-3269, https://doi.org/10.1016/j.biomaterials.2013.01.028.

4. Booth A.J., Hadley R., Cornett A.M., Dreffs A.A., Matthes S.A., Tsui J.L., Weiss K., Horowitz J.C., Fiore V.F., Barker T.H., Moore B.B., Martinez F.J., Niklason L.E., White E.S. Acellular normal and fibrotic human lung matrices as a culture system for in vitro investigation. Am J Respir Crit Care Med 2012; 186(9): 866-876, https://doi.org/10.1164/ rccm.201204-0754oc.

5. Bonenfant N.R., Sokocevic D., Wagner D.E., Borg Z.D., Lathrop M.J., Lam Y.W., Deng B., Desarno M.J., Ashikaga T., Loi R., Weiss D.J. The effects of storage and sterilization on decellularized and recellularized whole lung. Biomaterials 2013; 34(13): 3231-3245, https://doi.org/10.1016/j biomaterials.2013.01.031.

6. Kuevda E.V., Gubareva E.A., Sotnichenko A.S., Gumenyuk I.S., Gilevich I.V., Polyakov I.S., Porhanov V.A., Alekseenko S.N., Macchiarini P. Experience of perfusion recellularization of biological lung scaffold in rats. Russian Journal of Transplantology and Artificial Organs 2016; 18(1): 38-44, https://doi.org/10.15825/1995-1191-2016-1-38-44.

7. Wagner D.E., Bonenfant N.R., Sokocevic D., DeSarno M.J., Borg Z.D., Parsons C.S., Brooks E.M., Platz J.J., Khalpey Z.I., Hoganson D.M., Deng B., Lam Y.W., Oldinski R.A., Ashikaga T., Weiss D. Three-dimensional scaffolds of acellular human and porcine lungs for high throughput studies of lung disease and regeneration. Biomaterials 2014; 35(9): 2664-2679, https://doi.org/10.1016/j. biomaterials.2013.11.078.

8. O'Neill J.D., Anfang R., Anandappa A., Costa J., Javidfar J., Wobma H.M., Singh G., Freytes D.O., Bacchetta M.D., Sonett J.R., Vunjak-Novakovic G. Decellularization of human and porcine lung tissues for pulmonary tissue engineering. Ann Thorac Surg 2013; 96(3): 1046-1056, https://doi.org/10.1016/j.athoracsur.2013.04.022.

9. Kuevda E.V., Gubareva E.A., Sotnichenko A.S., Gilevich I.V., Gumenyuk I.S., Sharkova T.V., Karal-ogly D.D., Orlov S.V., Macchiarini P. Nonhuman lung decellularization: protocol optimization. Mezhdunarodnyy zhurnal prikladnykh i fundamental'nykh issledovaniy 2015; 8: 244-247.

10. Badylak S.F., Taylor D., Uygun K. Whole-organ tissue engineering: decellularization and recellularization of threedimensional matrix scaffolds. Annu Rev Biomed Eng 2011; 13 : 27-53, https://doi.org/10.1146/annurev-bioeng-071910-124743.

11. Kuevda E.V., Gubareva E.A., Krasheninnikov S.V., Grigoriev T.E., Gumenyuk I.S., Sotnichenko A.S., Gilevich I.V., Karal-ogly D.D., Orlov S.V., Chvalun S.N., Redko A.N., Alekseenko S.N., Macciarini P. Evaluation of the influence of decellularization on the changes in biomechanical properties of primate lungs. Doklady Biochemistry and Biophysics 2016; 470(1): 375-378, https://doi.org/10.7868/s0869565216300289.

12. Ruifrok A.C., Johnston D.A. Quantification of histochemical staining by color deconvolution. Anal Quant Cytol Histol 2001; 23(4): 291-299.

13. Bonvillain R.W., Danchuk S., Sullivan D.E., Betancourt A.M., Semon J.A., Eagle M.E., Mayeux J.P., Gregory A.N., Wang G., Townley I.K., Borg Z.D., Weiss D.J., Bunnell B.A. A nonhuman primate model of lung regeneration: detergent-mediated decellularization and initial in vitro recellularization with mesenchymal stem cells. Tissue Eng Part A 2012; 18(23-24): 2437-2452, https://doi.org/10.1089/ ten.tea.2011.0594. 
14. Gilpin S.E., Guyette J.P., Gonzalez G., Ren X., Asara J.M., Mathisen D.J., Vacanti J.P., Ott H.C. Perfusion decellularization of human and porcine lungs: bringing the matrix to clinical scale. J Heart Lung Transplant 2014; 33(3): 298-308, https://doi.org/10.1016/j.healun.2013.10.030.

15. Nichols J.E., Niles J., Riddle M., Vargas G.,
Schilagard T., Ma L., Edward K., La Francesca S., Sakamoto J., Vega S., Ogadegbe M., Mlcak R., Deyo D., Woodson L., McQuitty C., Lick S., Beckles D., Melo E., Cortiella J. Production and assessment of decellularized pig and human lung scaffolds. Tissue Eng Part A 2013; 19(17-18): 2045-2062. 